\title{
Optimum PI Controllers of Active Power Filters for Harmonic Voltage Mitigation in Multibus Industrial Power Systems
}

\author{
Josef Tlustý \\ Department of Power Engineering \\ Faculty of Electrical Engineering, CTU \\ Technická 2, 16627 Prague 6, Czech Republic \\ E-mail: tlusty@fel.cvut.cz
}

\author{
Viktor Valouch \\ Institute of Thermomechanics \\ Academy of Sciences of the Czech republic \\ Dolejškova 5, 18202 Praha 8, Czech Republic \\ E-mail: valouch@iee.cas.cz
}

\begin{abstract}
The bus voltage and branch current detection control strategies of the active power filters used in multibus power systems for harmonic voltage mitigation are analysed in terms of the optimum gains of PI controllers.
\end{abstract}

\section{Key words}

Active power filter, industrial power system, harmonic voltage mitigation, control strategies, optimum controllers.

\section{Introduction}

The performance and effectiveness of the active power filter (APF) depends on the point of its connection in the multibus power system [1]-[3].

The effectiveness of the bus voltage and branch current detection control strategies of the APFs used in multibus power systems is analysed and compared. The multiple harmonic optimization problem is formulated in such a way that its solution yields optimum parameters of PI controllers for both the methods.

\section{Control strategies}

The voltage and current detection feedback control strategies belong among control strategies very often used for parallel APF applied in simple power distribution systems. We proposed an application of these strategies in multibus industrial power systems with a few harmonic power sources [4]. The analysis was focused on obtaining basic knowledge about this new application of the strategies. The effectiveness of the connection of the APF at a specific bus may be assessed by the degree of the voltage harmonic mitigation at selected buses and by the demanded value of the APF current $I_{\text {IFRMs. }}$ But, the values of the controller gains should be taken into consideration as well with regard to problems with the stability of the whole control system.

\subsection{Voltage detection feedback control strategy}

The voltage detection feedback control strategy belongs among control strategies very often used for parallel APF applied in simple power distribution systems. We will analyse an application of this strategy in multibus power distribution systems with a few harmonic power sources.

Let the current vector $\boldsymbol{I}_{\mathrm{IF}}$ of APFs is generated by using a feedback of the node voltage vector

$$
\boldsymbol{I}_{\mathrm{IF}}=\boldsymbol{Y}_{\mathrm{G}} \boldsymbol{V}_{\mathrm{N}}
$$

where $\boldsymbol{Y}_{\mathrm{G}}$ is the feedback gain matrix.

\subsection{Current detection feedback control strategy}

This method is also used in simple power distribution systems, especially for mitigation of harmonic currents. It results, effectively, to the suppression of harmonic voltages too. Now, the feedback is specified by

$$
\boldsymbol{I}_{\mathrm{IF}}=\boldsymbol{G} \boldsymbol{I}
$$

where $\boldsymbol{G}$ is the feedback gain matrix and $\boldsymbol{I}$ is the branch current vector.

\section{Multibus industrial power system}

The system consists of overhead lines, cables, transformers, induction motors, rectifiers, and passive filters [4].

Let us suppose that a circuit has $n$ nodes and $b$ branches. The voltages $V_{\mathrm{N}}(i)$ at nodes are related to a reference node 0 , which voltage $V_{N}(0)$ is set to zero $\left(V_{\mathrm{N}}(0)=0\right)$. The vectors $\boldsymbol{V}_{\mathrm{I}}, \boldsymbol{I}_{\mathrm{I}}$ represent voltage and current sources (injected voltages and currents).

On the basis of the circuit equations we can obtain the matrix relation between $\boldsymbol{V}_{\mathrm{N}}$ and the injected voltages $\boldsymbol{V}_{\mathrm{I}}$ and currents $\boldsymbol{I}_{\mathrm{I}}$. Let us suppose that the vector $\boldsymbol{I}_{\mathrm{I}}$ of the $m$ currents injected consists of the vector $\boldsymbol{I}_{\mathrm{IL}}$ of $m_{\mathrm{L}}$ load currents and the vector $\boldsymbol{I}_{\mathrm{IF}}$ of $m_{\mathrm{F}}$ APF currents. The APF currents should compensate the node voltages $V_{\mathrm{N}}(i), i=1, \ldots, n$ induced by the injected load currents $\boldsymbol{I}_{\mathrm{IL}}$ and the voltages $\boldsymbol{V}_{\mathrm{I}}$

$$
\boldsymbol{V}_{\mathrm{N}}=\boldsymbol{Z}_{\mathrm{NIL}} \boldsymbol{I}_{\mathrm{IL}}+\boldsymbol{Z}_{\mathrm{NIF}} \boldsymbol{I}_{\mathrm{IF}}+\boldsymbol{H}_{\mathrm{NI}} \boldsymbol{V}_{\mathrm{I}}
$$


where $\boldsymbol{H}_{\mathrm{NI}}, \boldsymbol{Z}_{\mathrm{NI}}$ are the transfer matrices of the injected voltages and currents, $\boldsymbol{I}_{\mathrm{I}}=\left(\boldsymbol{I}_{\mathrm{IL}}, \boldsymbol{I}_{\mathrm{IF}}\right)^{\mathrm{T}}, \boldsymbol{Z}_{\mathrm{NI}}=\left(\boldsymbol{Z}_{\mathrm{NIL}}, \boldsymbol{Z}_{\mathrm{NIF}}\right)$,

$$
m_{\mathrm{L}}+m_{\mathrm{F}}=m \text {. }
$$

\section{Optimal control gain for multiple harmonic problem}

The solution of the single APF - single harmonic problem presented in [1] provides us with the set of optimal compensator currents for individual harmonic orders.

For the single APF - multiple harmonic problem the objective function may be written

$$
g_{\mathrm{H}}(f)=\sum_{h=2}^{H} \sum_{i=1}^{n-1} a_{i}^{h}\left|V_{\mathrm{N}}^{h}(i)\right|^{2}
$$

where the weighting factor $a_{i}\left(0 \leq a_{i} \leq 1, i=1, \ldots, n-1\right)$ reflects the different importance of the harmonic voltage mitigation at different nodes.

For the node voltage detection control strategy it holds for the $h$-th harmonic of the voltage at the node $i$

$$
\begin{gathered}
V_{\mathrm{N}}^{h}(i)=\frac{V_{\mathrm{N} 0}^{h}(i)+Y_{\mathrm{G}}(d)}{1-Y_{\mathrm{G}}(d) Z_{\mathrm{NIF}}^{h}(d)} . \\
\frac{\left[Z_{\mathrm{NIF}}^{h}(i) V_{\mathrm{N} 0}^{h}(d)-Z_{\mathrm{NIF}}^{h}(d) V_{\mathrm{N} 0}^{h}(i)\right]}{1-Y_{\mathrm{G}}(d) Z_{\mathrm{NIF}}^{h}(d)}
\end{gathered}
$$

where the current is injected into the node $f, d$ is the node where the voltage $V_{\mathrm{N}}^{h}(d)$ is detected, and

$$
\boldsymbol{V}_{\mathrm{N} 0}^{h}=\boldsymbol{Z}_{\mathrm{NIL}}^{h} \boldsymbol{I}_{\mathrm{IL}}^{h}+\boldsymbol{H}_{\mathrm{NI}}^{h} \boldsymbol{V}_{\mathrm{I}}^{h}
$$

is the node voltage vector without any compensation.

The optimum value $Y_{\mathrm{Gopt}}(d)$ may be determined by finding the minimum of $g_{\mathrm{H}}(f)(4)$ for $V_{\mathrm{N}}^{h}(i)$ declared by (5). For the trivial case of only one harmonic $h$ and one node $i$ an analytical solution can be found. For solving the general optimization problem ( $H$ harmonics and $n$ nodes) a proper numerical procedure is recommended.

For the branch current detection control strategy the voltages at the nodes $d_{1}, d_{2}$ and the current injected into the node $f$ may be expressed

$$
\begin{gathered}
V_{\mathrm{N}}^{h}\left(d_{1}\right)=V_{\mathrm{N} 0}^{h}\left(d_{1}\right)+Z_{\mathrm{NIF}}^{h}\left(d_{1}\right) I_{\mathrm{IF}} \\
V_{\mathrm{N}}^{h}\left(d_{2}\right)=V_{\mathrm{N} 0}^{h}\left(d_{2}\right)+Z_{\mathrm{NIF}}^{h}\left(d_{2}\right) I_{\mathrm{IF}} \\
I_{\mathrm{IF}}=G(d) Y^{h}(d, d)\left(V_{\mathrm{N}}^{h}\left(d_{1}\right)-V_{\mathrm{N}}^{h}\left(d_{2}\right)\right)
\end{gathered}
$$

where the branch current $I^{h}(d)$ is detected at the branch $d$ connecting the nodes $d_{1}, d_{2}$ and $Y^{h}(d, d)$ is the admittance of this branch. By using (7) and after some manipulation we obtain for the $h$-th harmonic of the voltage at the node $i$

$$
\begin{gathered}
V_{\mathrm{N}}^{h}(i)=V_{\mathrm{N} 0}^{h}(i)+Z_{\mathrm{NIF}}^{h}(i) I_{\mathrm{IF}}= \\
=\frac{V_{\mathrm{N} 0}^{h}(i)+G(d) Y^{h}(d, d)}{1-G(d) Y^{h}(d, d)\left(Z_{\mathrm{NIF}}^{h}\left(d_{1}\right)-Z_{\mathrm{NIF}}^{h}\left(d_{2}\right)\right)} \cdot \\
\cdot \frac{\left[Z_{\mathrm{NIF}}^{h}(i)\left(V_{\mathrm{N} 0}^{h}\left(d_{1}\right)-V_{\mathrm{N} 0}^{h}\left(d_{2}\right)\right)\right.}{1-G(d) Y^{h}(d, d)\left(Z_{\mathrm{NIF}}^{h}\left(d_{1}\right)-Z_{\mathrm{NIF}}^{h}\left(d_{2}\right)\right)}- \\
-\frac{\left.V_{\mathrm{N} 0}^{h}(i)\left(Z_{\mathrm{NIF}}^{h}\left(d_{1}\right)-Z_{\mathrm{NIF}}^{h}\left(d_{2}\right)\right)\right]}{1-G(d) Y^{h}(d, d)\left(Z_{\mathrm{NIF}}^{h}\left(d_{1}\right)-Z_{\mathrm{NIF}}^{h}\left(d_{2}\right)\right)}
\end{gathered}
$$

The optimum value $G_{\text {opt }}(d)$ may be determined by finding the minimum of $g_{\mathrm{H}}(f)(4)$ for $V_{\mathrm{N}}^{h}(i)$ declared by (8).

\section{Optimal PI controllers for multiple harmonic problem}

The optimal control gains $Y_{\mathrm{Gopt}}(d), G_{\mathrm{opt}}(d)$ for the multiple harmonic problem may be found by minimising the objective function (4) for $V_{\mathrm{N}}^{h}(i)$ declared by (5), (8), respectively. These gains should be applied for all harmonics of the selected range. The problem is formulated in the frequency domain, so all parameters and quantities, including the control gains, are complex values.

The node voltage and branch current detection control strategies in real systems are applied, on the contrary, in the time domain and work with a lot of harmonics that are present in time signals. For the branch current control strategy, for example, and for the PI controller of the APF, it holds

$$
I_{\mathrm{IF}}=G_{\mathrm{P}}(d) I(d)+G_{\mathrm{I}}(d) \int I(d) \mathrm{d} t
$$

where $G_{\mathrm{P}}(d), G_{\mathrm{I}}(d)$ are gains of the $P$ and $I$ components, and $I(d)$ is the current time response in the branch $d$. After the Laplace transform we can write

$$
I_{\mathrm{IF}}=\left[G_{\mathrm{P}}(d)+\frac{1}{s} G_{\mathrm{I}}(d)\right] \sum_{h} I^{\mathrm{h}}(d)
$$

and hence in the frequency domain

$$
I_{\mathrm{IF}}=\sum_{h}\left[G_{\mathrm{P}}(d)-\mathrm{j} \frac{G_{\mathrm{I}}(d)}{\omega_{\mathrm{h}}}\right] I^{\mathrm{h}}(d)
$$

where $I_{\mathrm{IF}}, I^{h}(d)$ are currents vectors (complex variables) now and $\omega_{\mathrm{h}}$ is the angular frequency of the $h$-th harmonic.

Thus, instead of searching for $Y_{\text {Gopt }}(d), G_{\text {opt }}(d)$, which are difficult to realize in the time domain for all harmonics by a simple controller, the gains of the PI 
controllers for both the control strategies should be found.

For the node voltage detection control strategy the objective function (4) can be used for $V_{\mathrm{N}}^{h}(d)$ declared by (5) if the complex value $Y_{\mathrm{G}}(d)$ is substituted by $Y_{\mathrm{GP}}$ (d) $-\mathrm{j} Y_{\mathrm{GI}}(d) / \omega_{\mathrm{h}}$.

For the branch current detection control strategy the same objective function (4) can be used for $V_{\mathrm{N}}^{h}(i)$ declared by (8) if the complex value $G(d)$ is substituted by $G_{\mathrm{P}}(d)-\mathrm{j} G_{\mathrm{I}}(d) / \omega_{\mathrm{h}}$.

A similar procedure can be applied for another type of the APF controller, while number of optimized control parameters may be higher than that in case of the PItype controller.

Let us analyze the results of the optimization procedure done for the different nodes $f(=2-6)$ where the APF current $I_{\mathrm{IF}}$ is injected. The results for the node voltage detection control strategy are summarized in Tab. 1, while for the branch current detection control strategy in Tab. 2. The tables show the THDs of the harmonic voltages $V_{\mathrm{N}}(i)$ at the nodes $i=1-6$, the total THD of the harmonic voltages at all these nodes (in p.u.system it is the square root of the objective function $g_{\mathrm{H}}(f)$ ), the respective value $I_{\text {IFoptRMs }}$ of the APF current injected into the nodes $f=2-6$, and the optimal parameters of the PI controllers. Let us consider that the node voltage $V_{\mathrm{N}}^{h}(d)$ is detected at the same node $d=f$ where the
APF is located, while the branch current $I^{h}(d)$ is detected at the branch $d$ lying upstream the node $f$.

Analyzing the results in Tab. 1, we can come to the conclusion that the node $f=3$ is likely the best one for the injection of the current $I_{\text {IFopt }}$. The requirements for THD $V_{\mathrm{N}}$, THD $V_{\mathrm{N}}(i), I_{\text {IFoptRMs }}$, and $Y_{\text {Gopt }}(d)$, which all should be as lowest as possible, are somewhat opposite one another, because higher values of $Y_{\text {Gopt }}(d)$ lead generally to lower THDs and $I_{\text {IFoptRMs. But very high }}$ gains may result to problems with the stability of the APF control strategy. Hence, a proper compromise should be done, considering actual demands of the real system.

Respecting the same rules, the node $f=6$ seems to be the best one in case of the branch current detection control strategy, Tab.2.

If we compare the data in Tab. 1 to those obtained in case of the repeated solution of the single harmonic problem [5] and thus for the different gains for individual harmonics, we see that the data in both the cases are practically the same. It means that by finding the optimum PI controller gains for the node voltage detection control strategy, which is very easy to realise, we may get near to the optimum obtained by the repeated solution of the single harmonic problem.

Table 1. Results of application of node voltage detection control strategy if multiple harmonic problem is solved for finding optimal PI controller parameters $Y_{\mathrm{GP}}, Y_{\mathrm{GI}}, d=f$

\begin{tabular}{||l||l|l|l||c||c|c|c|c|c|c||}
\hline \hline \multirow{2}{*}{ Node $f$} & \multirow{2}{*}{ THD $V_{\mathrm{N}}$} & \multirow{2}{*}{$I_{\mathrm{IFoptRMS}}$} & \multicolumn{2}{|c||}{$Y_{\mathrm{Gopt}}(d)$} & \multicolumn{6}{c||}{ THD $V_{\mathrm{N}}(i), i=1,2, \ldots, 6$} \\
\cline { 4 - 13 } & & $Y_{\mathrm{GP}}$ & $Y_{\mathrm{GI}}$ & 1 & 2 & 3 & 4 & 5 & 6 \\
\hline \hline 2 & 0.0266 & 1.2229 & 1.55 & 1892 & 0.0248 & 0.0056 & 0.0033 & 0.0008 & 0.0061 & 0.0031 \\
\hline 3 & 0.0264 & 1.1527 & 1.534 & 1796 & 0.0249 & 0.0052 & 0.0058 & 0.0014 & 0.0036 & 0.0005 \\
\hline 4 & 0.0408 & 1.0036 & 2.664 & 334.7 & 0.0253 & 0.0082 & 0.0096 & 0.0191 & 0.0172 & 0.0143 \\
\hline 5 & 0.0335 & 1.0519 & 0.689 & 535.5 & 0.0255 & 0.0053 & 0.0054 & 0.0081 & 0.0182 & 0.0042 \\
\hline 6 & 0.0302 & 1.09 & 1.399 & 780.4 & 0.0253 & 0.0049 & 0.0053 & 0.0044 & 0.0050 & 0.0133 \\
\hline \hline
\end{tabular}

Table 2. Results of application of branch current detection control strategy if multiple harmonic problem is solved for finding optimal PI controller parameters $G_{\mathrm{P}}, G_{\mathrm{I}}, d=$ branch upstream $f$

\begin{tabular}{||l|l|l|l|l||c|c|c|c|c|c||}
\hline \multirow{2}{*}{$\begin{array}{c}\text { Node } \\
f\end{array}$} & \multirow{2}{*}{ THD $V_{\mathrm{N}}$} & \multirow{2}{*}{$I_{\text {IFoptRMS }}$} & \multicolumn{2}{|c|}{$G_{\text {opt }}(d)$} & \multicolumn{6}{|c|}{ THD $V_{\mathrm{N}}(i), i=1,2, \ldots, 6$} \\
\cline { 4 - 11 } & & $G_{\mathrm{P}}$ & $G_{\mathrm{I}}$ & 1 & 2 & 3 & 4 & 5 & 6 \\
\hline \hline 2 & 0.0587 & 1.6619 & -3.639 & 66.22 & 0.0277 & 0.0217 & 0.0211 & 0.0286 & 0.0215 & 0.0220 \\
\hline 3 & 0.0557 & 1.1182 & -3.182 & 12.37 & 0.0260 & 0.0209 & 0.0210 & 0.0236 & 0.0230 & 0.0215 \\
\hline 4 & 0.0535 & 1.0835 & -1.192 & 0.184 & 0.0259 & 0.0175 & 0.0193 & 0.0132 & 0.0276 & 0.0241 \\
\hline 5 & 0.0519 & 1.1220 & -1.593 & 0.597 & 0.0263 & 0.0179 & 0.0179 & 0.0194 & 0.0263 & 0.0172 \\
\hline 6 & 0.0408 & 1.1491 & -1.681 & 0.726 & 0.0245 & 0.0128 & 0.0130 & 0.0142 & 0.0146 & 0.0178 \\
\hline
\end{tabular}


By comparing Tab. 2 to Tab. 1, we see that almost all data in Tab. 2 are higher than those in Tab. 1, but yet acceptable. Thus, we may find the proper PI controller gains for the branch current detection strategy too, even though the effectiveness of this strategy is lower than that of the node voltage detection control strategy.
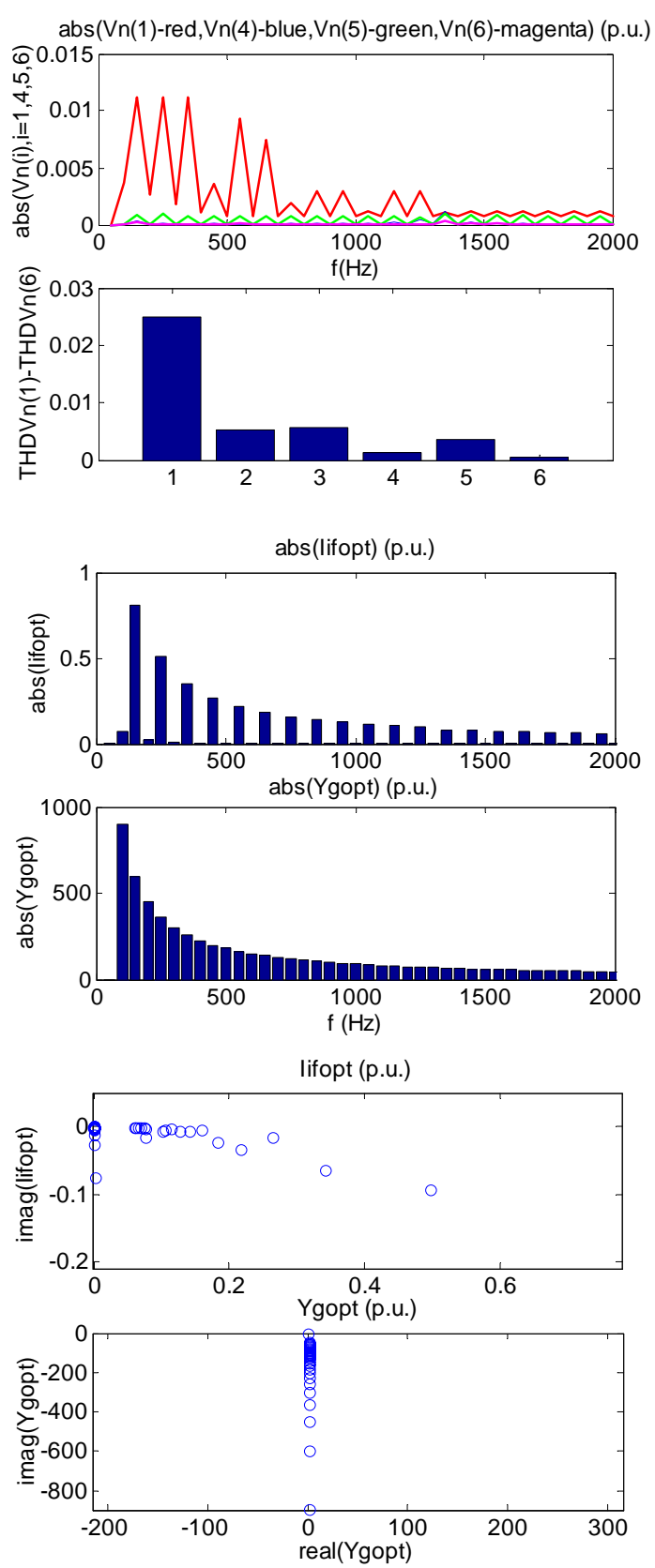

Fig. 1. APF is connected at bus 3 with the voltage feedback from bus 3

Fig. 1 shows the results of the analysis for the node voltage detection control strategy with $f=3$ (the location of the APF) and $d=3$ (the detection of the node voltage). Fig. 1a presents the harmonic spectra of the voltages at the buses $1,4,5$ and 6 and the THDs of the voltages at the buses 1-6. Fig. 1b shows the absolute values of the currents $I_{\text {IFopt }}$ and of the gains $Y_{\mathrm{Gopt}}$ for individual harmonics. Fig. 1c presents the values of these variables $I_{\mathrm{IFopt}}$ and $Y_{\mathrm{Gopt}}$ in the complex plane.
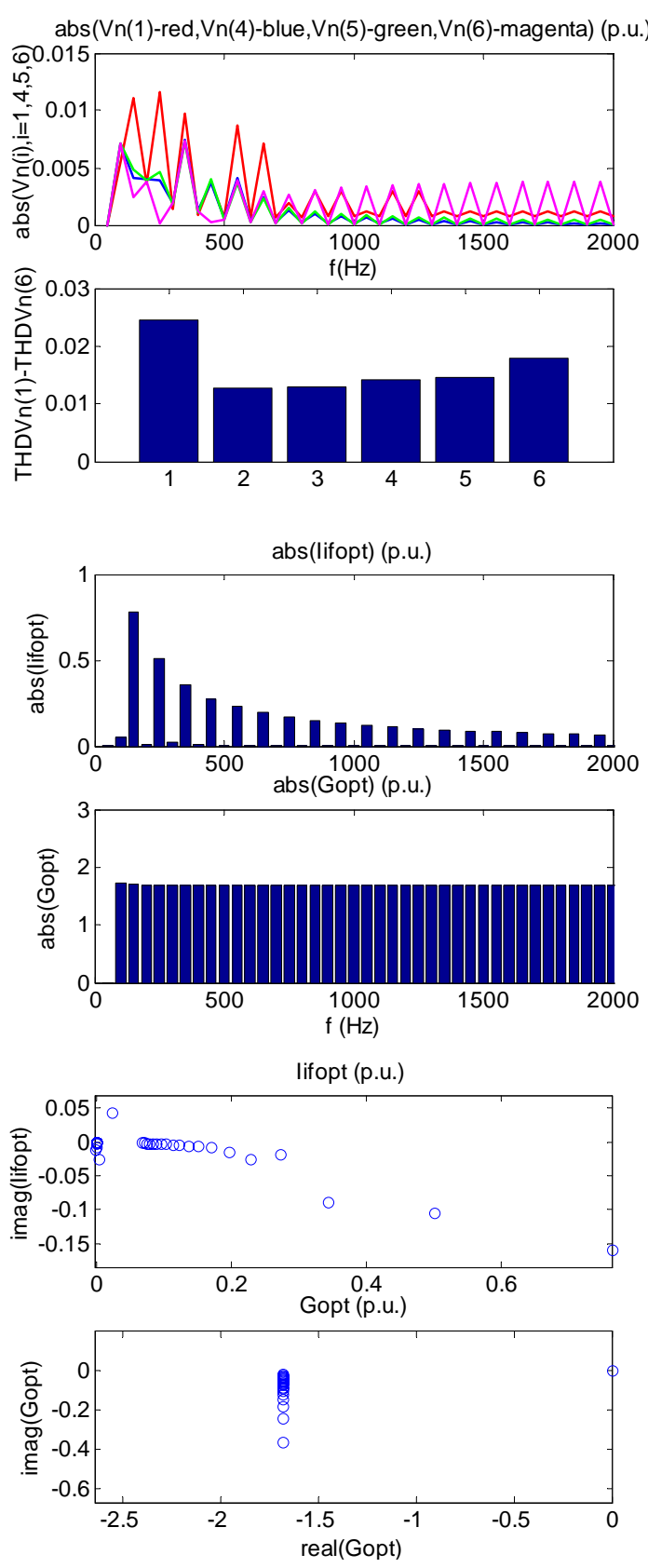

Fig. 2. APF is connected at bus 6 with the current feedback from branch 6 
Fig. 2 shows the results of the analysis for the branch current detection control strategy with $f=6$ and $d=6$.

\section{Conclusion}

The proposed formulation of the optimization problem for finding the optimum controller gains in both the control strategies represents a new effective way to achieve harmonic voltage

\section{References}

[1] Dai, X., Gretsch, R.: Optimal Compensator Currents for the Reduction of the Harmonic Distortion in Networks. Part 1: Analytic Solution. ETEP Vol. 4, No. 4, July/August 1994, pp. 301307.

[2] Gawlik, W.: Optimal Placement and Mutual Influence of Active Filters. $17^{\text {th }}$ Int. Conf. on Electricity Distribution (CIRED), 12-15 May, 2003, Barcelona, Spain, Session 2, pp.1-5.

[3] Morán, L.T.,Mahomar, J.J., Dixon, J.R.: Careful Connection. IEEE IA Mag., vol.10, no. 2, Mar/Apr 2004, pp. 43-50.

[4] Tlusty J., Valouch, V.: Connection of Shunt Active Power Filters in Multibus Industrial Power Systems for Harmonic Voltage Mitigation. Int. Conf. ICREPQ 05, Zaragoza, Spain, March 16-18 , 2005. mitigation in the buses of a multibus industrial power system. Although the proposed control strategies are easy applied in real systems, the obtained results are not far from those achieved by the application of the repeated solution of the single harmonic problem [5], which represents the most effective way to suppress the bus voltage harmonics, but it is rather difficult to realize, except the cases where only a few voltage harmonics should be compensated for.

[5] J. Tlusty, V. Valouch, P. Santarius, J. Skramlík, "Optimal Control of Shunt Active Power Filters in Multibus Industrial Power Systems for Harmonic Voltage Mitigation," Int. Conf. Electrimacs 05, Hammamet, Tunisia, April 17-20, 2005.

\section{Acknowledgement}

The financial supports of the Academy of Sciences of the Czech Republic (Institutional Research Plan Z20570509 of the Institute of Thermomechanics) and of the Ministry of Education, Youth and Sports (Research Plan MSM 6840770017 of the Czech Technical University) are highly acknowledged. 